
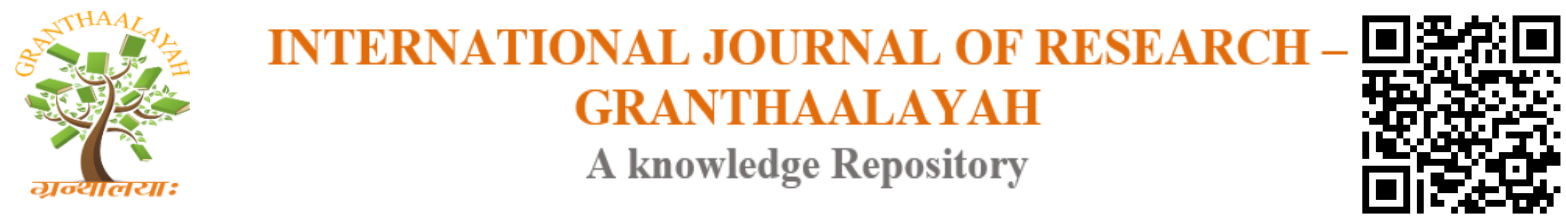

Science

\title{
ASSESSMENT OF PATHOLOGICAL CHANGE OF SCHISTOSOMA MANSONI INFECTIONIN THE LIVER USING ULTRASOUNOGRAPHY
}

\author{
Abdal Gadir Yonis ${ }^{1}$, Professor. Dr. Al Safi Ahmed Abdallash ${ }^{2}$, Dr.Mona Ahmed ${ }^{3}$, Dr. \\ Ashraf Mustafa Mohammed Osman 4 \\ ${ }^{1}$ Alshaab Teaching Hospital - Khartoum Sudan \\ ${ }^{2} \mathrm{MSc}, \mathrm{PhD}$, Professor of Radiology in College of Medical Radiological Sciences, Sudan \\ University \\ ${ }^{3} \mathrm{MSc}, \mathrm{PhD}$, Associate Professor in Diagnostic Radiology in college of medical radiological \\ sciences, Sudan University, Sudan \\ ${ }^{4}$ MBBSc, MSc, PhD, Consultant Sonologist in Sahiroon specialized Hospital \& Al Zaitoona
} Specialized Hospital

\begin{abstract}
Background: Schistosomiasis is a tropical parasitic disease caused by blood flukes of the genus Schistosoma, causes periportal fibrosis and portal hypertension in approximately 13\%-18\% of those afflicted with the disease in the Gezira irrigated areas of Sudan. This a prospective study compromise of 360 consecutive patients at North Gezira State between 2016 to 2019 aimed to assessment of pathological change in schistosomiasismansoni infection using B-mode grayscale ultrasound, The images done by portable Sono Scape- A6 machine using convex probe with frequency range from 3.5 to $5 \mathrm{MHz}$ and the data analyzed by SPSS version 15 . The result showed that the majority of patients were male and periportal fibrosis had high incidence associated with schistosomiasismansoni, Out of 360 patients with schistosomal PPF under study there were no PPF noted in $56(15.6 \%)$, while the remaining had mild in $106(29.4 \%)$, moderate in166 (46.1\%), and severe PPF in $32(8,9 \%)$ The study concluded that the B-mode Gray scale portable ultrasound machine is the most frequently used imaging modality to assess patients for the presence of periportalfibrosis especially in the endemic area.
\end{abstract}

Keywords: Portal Hypertension; B-Mode Ultrasound; Schistomiasis; Periportal Fibrosis and Portal Vein.

Cite This Article: Abdal Gadir Yonis, Professor. Dr. Al Safi Ahmed Abdallash, Dr.Mona Ahmed, and Dr. Ashraf Mustafa Mohammed Osman. (2019). "ASSESSMENT OF PATHOLOGICAL CHANGE OF SCHISTOSOMA MANSONI INFECTIONIN THE LIVER USING ULTRASOUNOGRAPHY." International Journal of Research - Granthaalayah, 7(7), 125-131. https://doi.org/10.29121/granthaalayah.v7.i7.2019.738. 


\section{Introduction}

Schistosomiasis is a tropical parasitic disease caused by blood flukes of the genus Schistosoma. 2 McManus DP, et al. (2014). By conservative estimates, at least 230 million people worldwide are infected with Schistosoma. Schistosomiasismansoni causes periportal fibrosis and portal hypertension in approximately $6 \%$ of all infected subjects.

The prevalence of Schistosomiasis in the Gezira irrigated areas of Sudan was lowered from 53.6\% in 1982 to $6.1 \%$ in 1989 by mass treatment with praziquantel under the Blue Nile Health project.During this same period Schistosomalperiportal fibrosis was found to affect $13-18 \%$ of those afflicted with the disease Ibrahim SZ et al 2008.

After the successful campaign of the Blue Nile Health Project in the eighties of the last century, the prevalence of Shistosomiasis is now again on the increase not only in selected endemic areas of Gezira, like Managil, where it reached 54.3\%, but also in Southern Blue Nile (32.6\%), Kassala province (45.2\%), and White Nile (35.5\%) A.Alla GN et al 2009.

The disease is mostly due to eggs deposited in host tissues by adult female worms which induce inflammatory and fibrotic lesions causing the development of significant pathology in the affected organs in host organs. The main lesion in hepatosplenicschistosomiasis is fibrosis in the portal tracts of the liver. The morbidity due to chronic Schistosomamansoniinfection In highly endemic areas, affects a significant proportion of the population. The disease mostly involves the colon, liver and spleen, with reactive splenic hyperplasia and hepatic fibrosis in the first stage, and later portal hypertension leading to a haemorrhage due to ruptured esophageal or gastric varices. . The significant and the goal of the study will help in limiting the severity of periportalfibrosis to avoid the complication by detecting it in early stage as well as to decreased the morbidity and mortality and the risk factors, Periportal fibrosis, which is the essential lesion, is generally seen after years of infection, but it has been found in children.Periportal thickening (PPT) or fibrosis is correlated with sonographicindicators of portal hypertension, namely reduced portal blood flow, portal systemic collaterals, dilatation of splenic vein, and splenomegaly. Li Y, et al. (2014). Diagnosis of schistosomiasis by imaging techniques has mostly focused on ultrasonography. Early use of grayscale ultrasound in the diagnosis of Schistosomiasismansoni, demonstrated lesions in the liver typical of schistosome-induced fibrosis like periportal fibrosis, which appeared as echogenic tubular shadows with anechoic lumen that radiated from the portahepatis. Grading of the severity of periportal fibrosis by ultrasonography in schistosomiasismansoni has been shown to correlate with disease burden Blanton RE.et al 2003.

Due to the reliability of US, this imaging method has been routinely used in the evaluation of hepatosplenicschistosomiasis for the past 30 years Azeredo LM, et al.2006 Lambertucci JR 2010 US has also been found to be valuable not only in the initial diagnosis but also in monitoring the regression of pathology following treatment with anti-schistosome drugs Lambertucci JR.et al2006 Williams GM, et al. 2000With the development of portable ultrasound equipment, this imaging technique became an acceptable tool in the assessment of organ morbidity due to schistosomiasis in the field setting. 


\section{Material and Methods}

This is a prospective study aimed to assessment of pathological change in schistosomiasismansoni infection used B-mode grayscale ultrasound, compromise of 360,56 patients have normal liver ,198 patients have periportal fibrosis with portal hypertension, and 106 patients have periportal fibrosis .carried out in the period from january2016 to 2019 in north Gazira state in Sudan and private clinic at Gebel Awlia Khartoum state. requested abdominal US was done with prior explanation of the investigation and informed written consent of the patients .ultrasound done was performed on SonoScape- A6 portable machine using convex probe with frequency range from 3.5 to $5 \mathrm{MHz}$ liver was scanned in various planes like transverse, sagittal, sub costal, obliqueinter costals and coronal planes. A comparative upper abdominal organ was done.

Various ultrasonographic features were observed, which included overall assessment of liver texture (periportal fibrosis), portal vein, splenic vein and splenic size.

These features were collected in a collection sheet(questionnaire), all questionnaires were daily checked from completeness and accuracy by investigator filled questionnaires were coded before entering data into computer using statistical package for social science (SPSS) version 15. Statistical analysis was performed using SPSS version 15.

\section{Results}

Table 1: Shows Gender distribution.

\begin{tabular}{|l|l|l|l|l|l|}
\hline \multicolumn{2}{|l|}{ Gender } & Frequency & Percent & Valid Percent & Cumulative Percent \\
\hline \multirow{3}{*}{ Valid } & Male & 244 & 67.8 & 67.8 & 67.8 \\
\cline { 2 - 6 } & Female & 116 & 32.2 & 32.2 & 100.0 \\
\cline { 2 - 6 } & Total & 360 & 100.0 & 100.0 & \\
\hline
\end{tabular}

Table 2: Shows Age Group distribution.

\begin{tabular}{|c|c|c|c|}
\hline \multicolumn{4}{|l|}{ Age Group } \\
\hline \multicolumn{4}{|c|}{\begin{tabular}{l|l|} 
Frequency & Percent $\mid$ Valid Percent $\mid$ Cumulative Percent \\
\end{tabular}} \\
\hline Valid $30-4077$ & 21.4 & 21.4 & 21.4 \\
\hline $40-50103$ & 28.6 & 28.6 & 50.0 \\
\hline $50-60121$ & 33.6 & 33.6 & 83.6 \\
\hline $60-7053$ & 14.7 & 14.7 & 98.3 \\
\hline $70-806$ & 1.7 & 1.7 & 100.0 \\
\hline \begin{tabular}{|l|l|} 
Total & 360 \\
\end{tabular} & 100.0 & 100.0 & \\
\hline
\end{tabular}




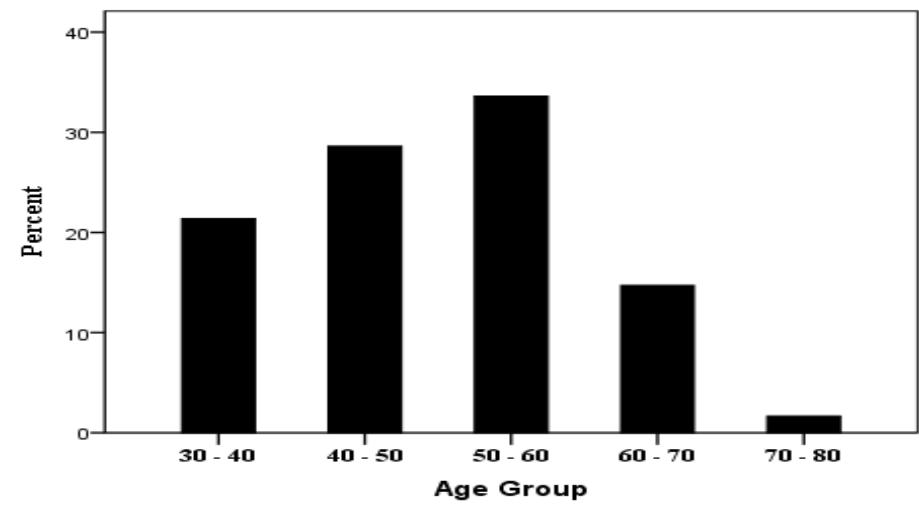

Figure 1: 2-D bar chart shows Age Group distribution.

Table 3: distribution of PPF thickness

\begin{tabular}{|l|l|l|l|l|l|}
\hline \multicolumn{2}{|c|}{} & Frequency & Percent & Valid Percent & Cumulative Percent \\
\hline & Total & 360 & 100.0 & 100.0 & \\
\hline Valid & None & 56 & 15.6 & 15.6 & 15.6 \\
\hline & Mild & 106 & 29.4 & 29.4 & 45.0 \\
\hline & Moderate & 166 & 46.1 & 46.1 & 91.1 \\
\hline & Severe & 32 & 8.9 & 8.9 & 100.0 \\
\hline
\end{tabular}

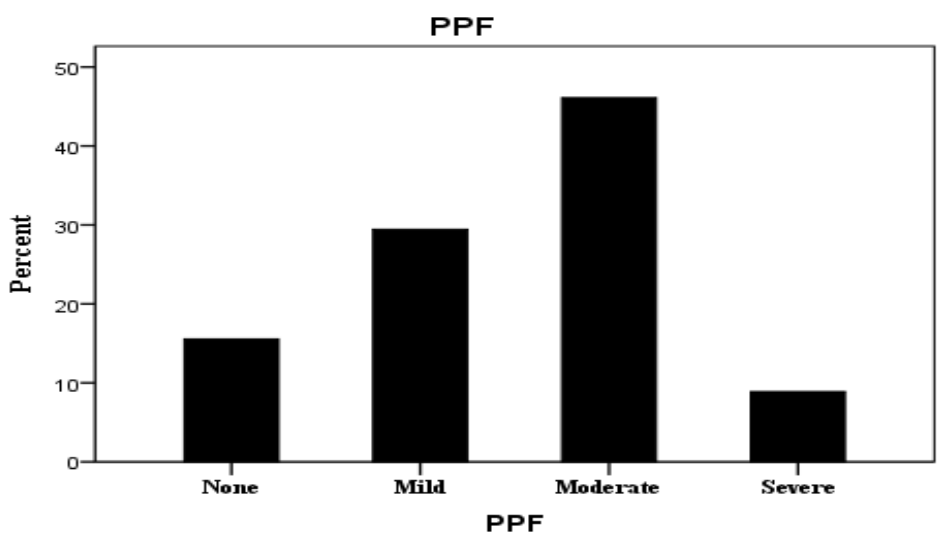

Figure 2: 2-D bar chart represents the distribution of PPF thickness

Table 4: cross-tabulation table show the relation between of the mean PV Width $(\mathrm{cm})$ SV Width $(\mathrm{cm})$ Spleen size $(\mathrm{cm})$ and group control normal and Patients group PPF and PHT.

\begin{tabular}{|l|l|l|l|}
\hline & variable & N & Mean \\
\hline PV Width (Cm) & Normal & 56 & 1.03 \\
\cline { 2 - 4 } & PPF & 106 & 1.25 \\
\cline { 2 - 4 } & Portal Hypertension & 198 & 1.52 \\
\hline SV Width (Cm) & Normal & 56 & .63 \\
\cline { 2 - 4 } & PPF & 106 & .887 \\
\cline { 2 - 4 } & Portal Hypertension & 198 & 1.12 \\
\hline Spleen size (Cm) & Normal & 56 & 10.02 \\
\cline { 2 - 4 } & PPF & 106 & 12.68 \\
\cline { 2 - 4 } & Portal Hypertension & 198 & 17.37 \\
\hline
\end{tabular}




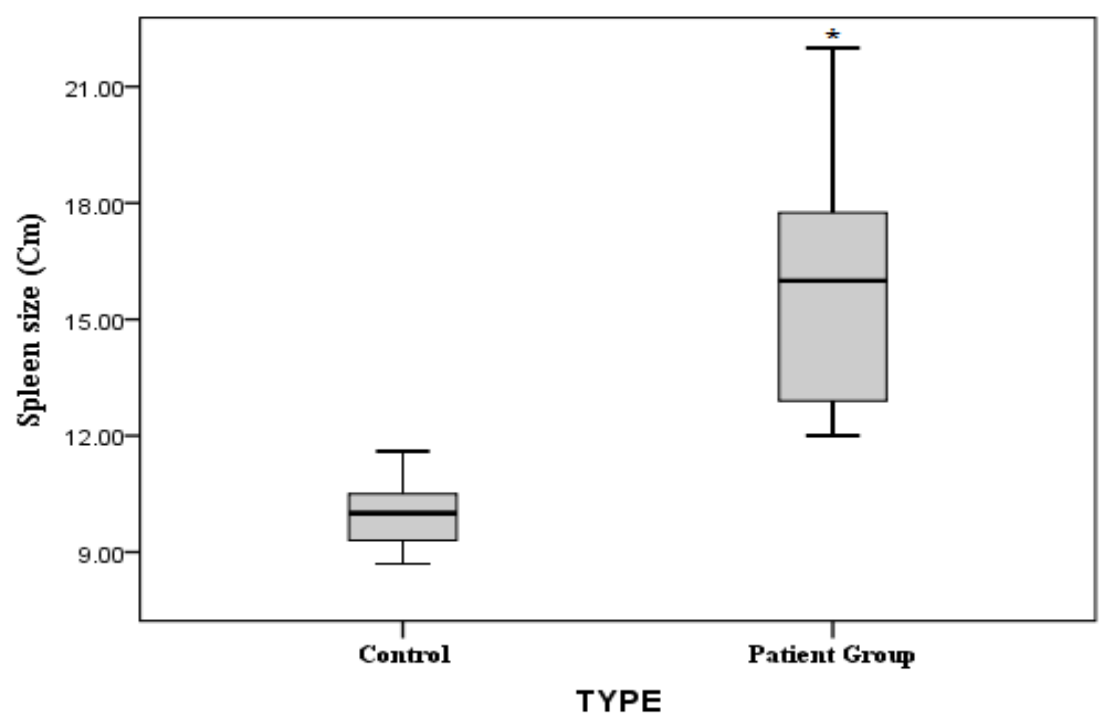

Figure 3: the mean of spleen size in control and Patients group. The strike indicates significant difference $(P<0.05)$.

\section{Discussion}

The data of this study consist of 360 case, 244 patients were mostly male forming the incidence of $67.8 \%$ table (1) because in retract with environment of schistosomiasis. This record agreewith Safaa. A 2017 whofound more incidence and more severity in male. This study found patients in age between 30 to 80 years distribution were mostly in (50-60)years forming the incidence of $33.6 \%$ (121)patient, in(40-55)years 28.6\% (103)patients table(2). This study was used for both diagnosis and grading of periportal fibrosis .Grading was allotted this grades by modification of the original method described by Safaa. A 2017 which was conducted in the same institution .Grade o none,gade1 mild, grade $\mathbf{1 1}$ moderate to severe and grade III advance severe. Out of 360 patients with schistosomal PPF under study there were no PPF noted in 56(15.6\%), while the remaining had mild in 106 (29.4\%), moderate in166 (46.1\%), and severe PPF in 32(8,9\%) table(3).The $29.4 \%$ of patient not a percentage, because It is important not to limit cases recorded as positive for periportal fibrosis to those where ultrasound examination reveals pathlogical changes unequivocally. The detection of mild pathology is not important in clinical practice, since patients with .borderline. abnormalities have never been observed to suffer complications such as variceal bleeding. The range of borderline pathology is large, which is not surprising, since schistosomiasis is a progressive disease. Cut-off points at which borderline findings are considered abnormal were proposed. This is a Significant of the study. This mean that the schistosomiasismansoni in the endemic area is play a key role in causes of periportal fibrosis and PHT. This was in accordance with those previous studies which were recorded the result of PHT and PPF like whomBurchardet al.1998 concluded that PPF mainly due to schistosomiasis. Of this study sonographic visualization of the portal vessels were obtained in most cases in patient with schistosomiasis were significantly greater when compare with the mean obtained for in individual, and in the normal control group. The increase in the width of the splenic vein was impressive, its mean that the width in patient with PHT was twice the width measured in the control group because the splenic vein don't effected of collateral while the portal vein lack of the sensitivity due to collateral path way which partially decompress the system. The upper limit of the normality for 
the mean width of portal was set through $1.03 \mathrm{~cm}$ for the portal vein $0.63 \mathrm{~cm}$ for the splenic vein width. The mean width over $1.25 \mathrm{~cm}$ and $1,52 \mathrm{~cm}$ for the portal vein, and $0.89 \mathrm{~cm}$ and $1,12 \mathrm{~cm}$ for the splenic vein in group patient (periportal fibrosis + portal hypertentesion) were $\% 100$ specific for the diagnosis of portal hypertension. (Table 4). The upper limit of normality for the width of the portal vessels has not yet firmest established for the portal vein, for example some author have chosen 12mmCerri ,G 1984 while other suggested 20mm Weill ,F.S 1982. Indicated that the splenic veins the best discriminated vessels for the diagnosis of PHT this agree with the research of Zoliet al 1985 which were recorded that the spleen vein was) (sensitivity of $85 \%$ and specifically $96 \%$ followed by the portal vein sensitivity $76 \%$ and specifically of $90 \%$ ).

The upper limit of the normality for the mean width of spleen size was set through $10.02 \mathrm{~cm}$.In group patient The mean width of spleen size over $12.68 \mathrm{~cm}$ for PPF patient and $17.4 \mathrm{~cm}$ for PHT patient In figure $(2)$ The strike indicate significant difference $(P<0.05)(014)$ on anther hand the enlarged spleen is low specifically according to deferent sears like acute involvement of the spleen such as in malaria and anemia .This was in accordance with the study of $\mathbf{C}$. Bru,J. Bosch et al 1998 which showed that the spleen dimension is the US sign most commonly associated to the presence of PHT contrarily to other sign its sensitivity is high, while is ranges 50_60\% according to different series it's an independent predictor of esophageal varies.

The study concluded that the portable ultrasound machine is the most frequently used imaging modality to assess patients for the presence of periportal fibrosis especially in the endemic area because its noninvasive methods and simple technique.

\section{References}

[1] A.Alla GN. Results of the 2005 surveillance project in Gezira, Southern Blue Nile, Kassala, and White Nile provinces. National schistosomiasis\& STH control program, Federal Ministry of Health. Personal Communication 2009

[2] Marinho CC, Voieta I, Azeredo LM, et al. (2006) Clinical versus ultrasound examination in the evaluation of hepatosplenicschistosomiasismansoni inendemic areas. MemInstOswaldo Cruz, Rio de Janeiro101: 317-321.

[3] King CH, Magak P, Salam EA, Ouma JH, Kariuki HC, Blanton RE. (2003) Measuring morbidity in schistosomiasismansoni: relationship between imagepattern, portal vein diameter and portal branch thickness in large-scale surveysusing new WHO coding guidelines for ultrasound in schistosomiasis. TropicalMedicine \& International Health 8: 109-117

[4] G.-D.Burchardet al. Ultrasound and chemical markers of hepatosplenic schistosomiasis1998

[5] Ibrahim SZ, FRCS, Shah T, FRCS, Arbab BM, MRCP, Abdel-Wahab O, DMRD2008Risk factors for bleeding in patients with asymptomatic oesophagealvarices secondary to schistosomal portal hypertension: a longitudinal hospital based study .subclinical disease and fibrosis marker measurements. Trans R Soc Trop Med Hyg 94: 191-197.

[6] Pinto-Silva RA, Queiroz LC, Azeredo LM, Silva LC, Lambertucci JR (2010) Ultrasound in schistosomiasismansoni. MemInstOswaldo Cruz 105: 479-4

[7] Cota GF, Pinto-Silva RA, Antunes CMF, Lambertucci JR. (2006) Ultrasound and clinical investigation of hepatosplenicschistosomiasis: evaluation of splenomegaly and liver fibrosis four years after mass chemotherapy with oxamniquine. The American Journal of Tropical Medicine and Hygiene74:103-107. 
[8] Olveda DU, Olveda RM, Lam AK, Chau TNP, Li Y, et al. (2014) Utility of Diagnostic Imaging in the Diagnosis and Management of Schistosomiasis. Clin Microbial 3: 142. doi:10.4172/23275073.1000142

[9] Olveda DU, Li Y, Olveda RM, Lam AK, McManus DP, et al. (2014) Bilharzia in the Philippines: past, present, and future. Int J Infect Dis 18: 52-56.

[10] Li YS, Sleigh AC, Ross AG, Li Y, Williams GM, et al. (2000) Two-year impact of praziquantel treatment for Schistosomajaponicum infection in China: reinfection,

[11] Safaa. A 2017 Study of Liver in Bilharziasis Patients by ultrasound using Image Pattern Recognitions Sudan.

*Corresponding author.

E-mail address: ashrafmerafabi@ gmail.com 